\title{
Statistical discrimination of controls, schizophrenics, depressives and alcoholics using local magnetoencephalographic frequency-related variables
}

\author{
Fehr T., Wienbruch C., Moratti S., Rockstroh B., and Elbert T. \\ University of Konstanz, Konstanz, Germany
}

\section{Introduction}

Atypically enhanced activity in the delta and theta EEG frequency bands has frequently been reported for schizophrenic patients, while alpha activity is often attenuated in these patients $[2,9,10,12]$. MEG and EEG data provide an advanced approach to analyze complex brain functioning and to examine differences between different psychiatric patient groups due to their brain activity. Past analyses using different physiological parameters to discriminate a psychiatric patient group from controls reached statistical correct classification rates of at maximum 80 percent. Results usually shifted to chance when adding a third group to the analysis. Winterer (2000) [13], for example, could discriminate between schizophrenic patients and controls with a correct classification rate of 77 percent when using delta power, signal power at $\mathrm{Cz}$ and power values of the high alpha range as variables in a discriminant analysis. Including a group of depressive patients in the analysis reduced the correct classification rate to 50 percent. Gallhofer (1991) [5] used 50 topographical frequency-related EEG-parameters in a discriminant analysis with schizophrenic and depressive patients and controls. He classified 49 out of the 50 subjects correctly.

Strategies that try to describe the physiological substrate of psychiatric diseases with only a few parameters possibly over-simplify the nature of the phenomenon [see also 5]. More complex strategies are possibly more adequate to describe complex phenomena such like psychiatric diseases.

The present study examined to what extent delta-, thetaand alpha-band-related source space activity can separate controls, schizophrenics, depressives and alcoholics by discriminant analysis. The analyses are meant as a first step towards an evaluation of a set of physiological parameters that could possibly be representative of certain psychiatric gross groups. In order to explore possible methods sensitive to these physiological parameters, different strategies of MEG source space analysis and statistical procedures were performed on data obtained during three different mental modalities (rest, mental calculation and mental imagery). Enhancement in focal [1] as well as in multiple [4] slow wave activity has been reported for schizophrenic patients. A reduction of alpha activity has been reported for schizophrenic [10,12] and alcohol [3] patients as well. For the analysis of focal sources we performed the dipole density method that has been shown as a valid tool in the vicinity of the detection of pathological attributed slow wave activity for example around tumors [8] or lesions
[11]. Multiple source activity in the slow wave and alpha range was detected by the minimum-norm method [6,7].

\section{Method}

30 schizophrenic (predominantly paranoid or disorganized schizophrenia, 12 female, mean age 31.1 \pm 8.6 years, 25 right-handed, 5 left-handed, 24 medicated, 6 unmedicated), 10 depressive ( 7 female, mean age $47.5 \pm 7.6$ years, 9 right-handed, 1 left-handed, 8 medicated, 2 unmedicated) and 12 alcoholic patients (1 female, mean age 39.7 \pm 10.9 years, 11 right-handed, 1 left-handed) and 18 healthy controls ( 2 female, mean age $31.7 \pm 12.4$ years, all right-handed) served as subjects during a resting, a mental calculation and a mental imagination condition (each in a 5 minute epoch).

In the resting period, subjects were asked to relax but stay awake and not to engage in any specific mental activity; in the mental arithmetic period, subjects were asked to translate the words of a common German folksong letter by letter into numbers ('a' corresponding to $1, b^{\prime}=2,{ }^{\prime} c^{\prime}=3$ etc.) and total them up; in the mental imagery condition, subjects were asked to imagine as vividly as possible walking a well-known and recently strolled footpath, e.g. through the hospital area.

Data were obtained from magnetoencephalographic recordings (148-channel whole-head neuromagnetometer, MAGNES $^{\circledR}$ WH 2500, 4D Neuroimaging, San Diego, USA) with a $678.17 \mathrm{~Hz}$ sampling rate, using a band-pass filter of 0.1-200 Hz. Subjects were asked to fixate on a colored mark on the ceiling of the chamber in order to avoid eye- and head-movement. For artifact control, eye movements (EOG) were recorded from four electrodes attached to the left and right outer canthus and above and below the right eye. The electrocardiogram (ECG) was monitored via electrodes attached to the right collarbone and the lowest left rib.

For each of the measured epochs the data were band-pass filtered in the delta $[1.5-4.0 \mathrm{~Hz}]$, theta $[4.0-8.0 \mathrm{~Hz}]$ and alpha [low: 8.0-10.5 Hz; high: 10.5-13.0 Hz] band, and the number of sample points was reduced by a factor of 16 prior to further source analyses.

The multiple source activity was located employing sources by means of the minimum-norm (MMN) estimate (L2-norm) [6,7] for the delta, theta and alpha range. Two strategies were realized: MN1) Over all data time points with a global field power between 3000 and 18000 [ft] that did not correlate with a prominent eye-blink pattern a MMN solution was calculated. The solutions were then averaged over all time points; MN2) Emphasis on commonly occurring topographies (identified by a sepa- 
rate correlational analysis - reported elsewhere - , only for the delta and theta range): the analysis was repeated, using only the 20 time points (topographies) with the strongest GFP. The resulting MMN-Maps of both strategies MN1 and MN2 were then divided in 10 regions (see figure 2).

Focal slow wave activity was determined by the dipole density method (DD) for the delta and theta range. Artifact-free time segments were determined by visual inspection. Single equivalent current dipoles in a homogeneous sphere were fitted for each time point in the selected epochs. Only dipole fit solutions at time points with a root mean square 100 fT < $\left(\mathrm{RMS}=\left(\sqrt{ }\left(1 / \mathrm{n} \Sigma\left(\mathrm{x}_{\mathrm{i}}\right)^{2}\right)\right)<300 \mathrm{fT}\right.$ and with a goodness of fit (GOF) greater than 0.90 were accepted for further analysis. These restrictions should ensure that neither artifacts nor small amplitude biological noise would affect the results, and that only dipolar fields that were generated by focal sources were analyzed. The percentage of dipoles fitted per second in a particular area was submitted to the statistical analyses. The source space data of the DD were divided into 10 voxels, five in each hemisphere: prefrontal, frontal, temporal, parietal and occipital.

Standard discriminant analyses were performed separately for the slow wave related source values of each model (DD, MN1 and MN2) and condition (20 regional source variables due to the delta and theta range). In a second step all the models were added up by the multiple alpha source values (alpha low and high, each band 10 regional source variables) estimated by the MN1 strategy. In a third step the amount of variables in each discriminant model was stepwise reduced oriented on the partial significance levels of the variables. The reduction ended at that point when the models became worse (due to their correct classification rates).

\section{Results}

In a first step 20 frequency related regional source variables (for the delta and theta frequency range) were included in discriminant analyses for the different models (DD, MN1 and MN2, see methods) separately for each condition. The discriminant functions for the DD method did not reach significance (see table 1 for the results in detail) for all conditions. The discriminant function for the MN1 and the MN2 methods reached significance for the rest and the mental calculation condition and showed a trend for the imagination condition (see table 1). The different groups could be separated comparably well with a correct overall classification rate about 70 percent.

In a second step all models were added up by 20 regional source space variables of the lower and higher alpha band (model MN1, see methods). For the DD slow wave variables in combination with the $20 \mathrm{MN} 1$ alpha variables the discriminant function for the rest condition was significant (see table 1 for the results in detail). For the multiple slow wave variables (MN1 as well as MN2) in combination with the $20 \mathrm{MN} 1$ alpha variables all discriminant functions were significant (see table 1). The overall correct classification rates of the calculated
Discriminant Analyses

\begin{tabular}{cccccc}
\hline \multicolumn{5}{c}{ Discriminant Analyses } \\
\hline Step 1 - delta and theta band (each 10 regions) \\
\hline \multirow{3}{*}{ DD } & Condition & Lambda & F & p & \% correct \\
& rest & n.s. & n.s. & n.s. & 70 \\
& arit & n.s. & n.s. & n.s. & 70 \\
& imag & n.s. & n.s. & n.s. & 64 \\
\hline \multirow{3}{*}{ MN1 } & rest & .20 & 1.7 & .006 & 79 \\
& arit & .23 & 1.5 & .03 & 74 \\
& imag & .25 & 1.4 & .06 & 71 \\
MN2 & rest & .20 & 1.7 & .008 & 76 \\
& arit & .24 & 1.4 & .04 & 76 \\
& imag & .25 & 1.6 & .06 & 71 \\
\hline \hline
\end{tabular}

\begin{tabular}{cccccc}
\hline \hline \multicolumn{5}{c}{ Step 2 - delta, theta and alpha ${ }^{*}$ band (each 10 regions) } \\
\hline Model & Condition & Lambda & F & p & \% correct \\
\hline \multirow{3}{*}{ DD } & rest & .03 & 1.6 & .009 & 94 \\
& arit & n.s. & n.s. & n.s. & 96 \\
& imag & n.s. & n.s. & n.s. & 90 \\
\hline \multirow{3}{*}{ MN1 } & rest & .02 & 1.8 & .003 & 99 \\
& arit & .02 & 1.7 & .005 & 94 \\
& imag & .02 & 1.7 & .005 & 94 \\
\hline \multirow{3}{*}{ MN2 } & rest & .03 & 1.5 & .03 & 93 \\
& arit & .03 & 1.6 & .02 & 91 \\
& imag & .02 & 1.8 & .002 & 94 \\
\hline
\end{tabular}

Tab. 1: results of the discriminant analyses (first root of the discriminant function) for the different combinations of the source related variables (see text); $D D=$ dipole density, MN1, MN2=minimum-norm strategy 1 and 2 (see text); *) alpha low and alpha high were calculated only for MN1 and added to the slow wave related source variables of the models DD, MN1 and MN2.

discriminant functions ranged between 90 and 99 percent. In a third step for all discriminant models the amount of included variables were stepwise reduced. Only one discriminant model could be improved by cancelation of variables.

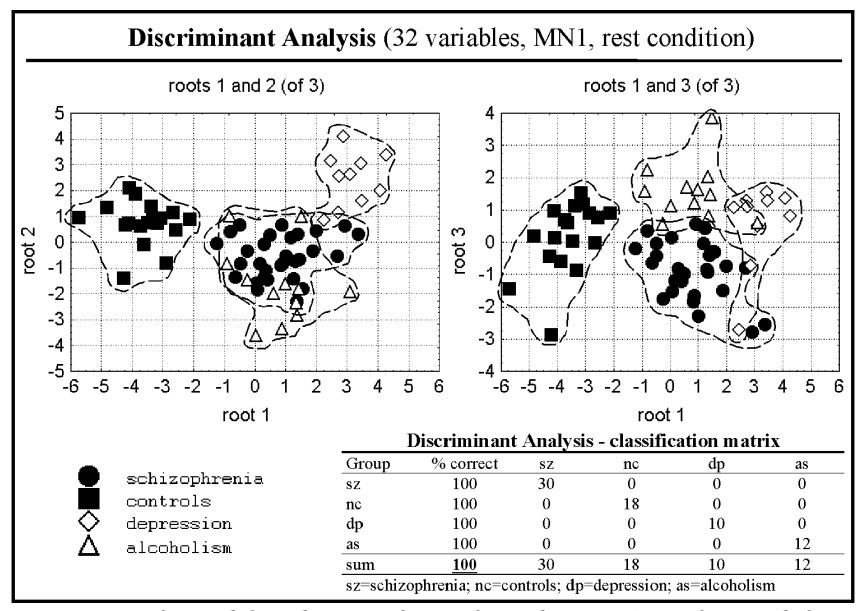

Fig. 1: plotted loadings of single subjects (see legend for group identification) on the different roots of a discriminant function (1st vs 2 nd root and 1 st vs 3 rd root) including 32 frequency band related source variables (model MN1, see text) and the corresponding classification matrix.

A stepwise reduction of variables led to a model with 32 variables estimated by the minimum-norm method (in the rest condition, model $\mathrm{MN1}$ ) that discriminated all three patient groups and the controls with a correct classification rate of 100 percent (see table 2 and figure 
1). All regional delta (mainly left prefrontal, temporal and left occipital) and theta (mainly left prefrontal, left and right temporal and right occipital) and some alpha low (mainly frontal and right prefrontal) and alpha high (mainly frontal, occipital and right temporal) source space variables were necessary to reach 100 percent correct classification and a maximum of significance of the discriminant function (see figure 2).

Stepwise Discriminant Analysis

Step 3-32 variables out of delta, theta and alpha bands

\begin{tabular}{cccccc} 
Model & Condition & Lambda & F & p & \% correct \\
\hline MN1 & rest & .03 & 2.4 & $<.0001$ & 100 \\
\hline
\end{tabular}

Tab. 2: results of a stepwise discriminant analysis (first root of the discriminant function) for 32 source related variables of model MNI (see text).

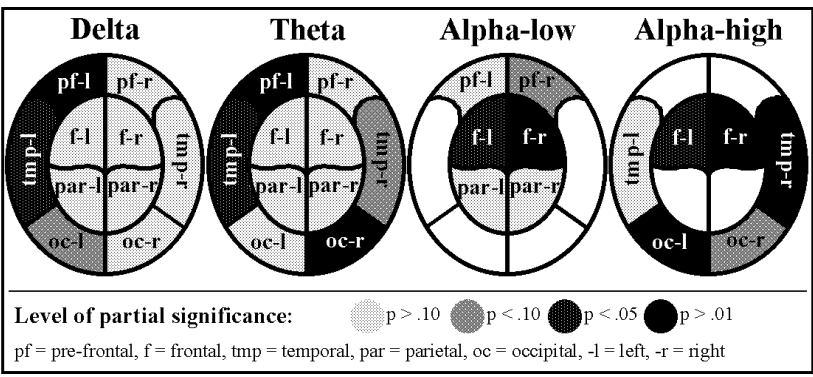

Fig. 2: schematic illustration of the frequency band related source variables of model MNI (see text) in a stepwise reduced discriminant analysis (significance levels of the partial lambdas of the variables are indicated by different shadings).

\section{Discussion}

The results showed that it was possible to discriminate different patient groups successfully with the highest accuracy when using a network of spatial (in form of different locations) and functional (in relation to different frequency generators) information. Furthermore the results provided a promising basis for future development and application of functional magnetic source imaging in psychopathological and -diagnostical domains. However the results of the discriminant analyses performed in the present study have to be interpreted carefully because of the relatively small subject samples. The results should be replicated with bigger patient groups. A next step should be the differentiation of the concept in the perspective of the development of subgroup specific feature detection. For schizophrenic patients a statistical discrimination between patients with predominantly positive or negative symptoms could be interesting, as well as other diagnostical aspects. The time course of such physiological variables examined in this study would be another important aspect that has to be considered carefully in further research.

The present explorative work offers a set of physiological variables that could be hypothetically tested in different patient groups and provides a promising toolbox of strategies for analyzing frequency-related data on the basis of functional magnetic source imaging.

\section{References}

[1] Canive, J.M., Lewine, J.D., Edgar, J.C, Davis, J.T., Miller, G.A., Torres, F., Tuason, V.B. (1998): Spontaneous brain magnetic activity in schizophrenic patients treated with aripiprazole. Psychopharmacology Bulletin 34:101-105.

[2] Elbert, T., Lutzenberger, W., Rockstroh, B., Berg, P., Cohen, R. (1992): Physical aspects of the EEG in schizophrenics. Biological Psychiatry 32: 595-606.

[3] Enoch, M.A., White, K.V., Harris, C.R., Robin, R.W., Ross, J., Rohrbaugh, J.W., Goldman, D. (1999): Association of low voltage alpha EEG with a subtype of alcohol use disorders. Alcoholism: Clinical and Experimental Research 23(8): 13121319

[4] Fehr, T., Kissler, J., Elbert, T., Wienbruch, C., Moratti, S., Rockstroh, B. (2001): Source distribution of neuromagnetic focal slow waves and MEG-delta activity in schizophrenic patients. Biological Psychiatry (in press).

[5] Gallhofer, B., Malle, B., Körner, E., Wieselmann, G., Kunz, S. (1991): EEG-Mapping bei Schizophrenie und Depression ein multivariater Zugang. Fortschr.-Neurol.-Psychiatr. 59: 447452.

[6] Hämäläinen, M., Ilmoniemi, R.J. (1984): Interpreting Measured Magnetic Fields of the Brain: Estimates of Current Distributions. Technical Report TKK-F-A559, 1984

[7] Hauk, O., Berg, P., Wienbruch, C., Rockstroh, B., Elbert, T. (1999): The minimum norm method as an effective mapping tool for MEG analysis. In: Yoshimoto, T., Kotani, M., Kuriki, M., Karibe, H., Nakasato, N., editors. Recent advances in biomagnetism (Proceedings of the 11th conference on biomagnetism). Tohoku University Press, Sendai 1999, pp. 213216.

[8] Lewine, J.D., Orrison, W.W. (1995): Magnetoencephalography and Magnetic Source Imaging. In: Orrison, W.W., Lewine, J.D., Sanders, J.A., Hartshorne, M.F., editors. Functional Brain Imaging. Mosby-Year Book, Inc., St- Louis 1995, pp. 404

[9] Rockstroh, B., Elbert, T., Berg, P. (1997): Die Untersuchung von Strukturen und Funktionen des Gehirns in der experimentellen Psychopathologie am Beispiel der Schizophrenien. In: Rockstroh, B., Elbert, T., Watzl, H., editors. Impulse für die Klinische Psychologie. Göttingen, Hogrefe: 1-29.

[10] Shagass, C. (1991): EEG studies of schizophrenia. In: Steinhauer, S.R., Gruzelier, J.H., et al., (Eds.). Neuropsychology, Psychophysiology, and Information Processing. Handbook of Schizophrenia. Amsterdam, Elsevier, pp. 39-69.

[11] Vieth, J.B., Kober, H., Grimmich, P. (1996): Sources of spontaneous slow waves associated with brain lesions, localized by using the MEG. Brain Topography 8(3): 215-221

[12] Winterer, G., Herrmann, W.M. (1995): Über das Elektroenzephalogramm in der Psychiatrie: Eine kritische Bewertung. Zeitschrift für Elektroenzephalographie, Elektromyographie und verwandte Gebiete 26: 19-37.

[13] Winterer, G., Ziller, M., Dorn, H., Frick, K., Mulert, C., Wuebben, Y., Herrmann, W.M. (2000): Frontal dysfunction in schizophrenia - a new electrophysiological classifier for research and clinical applications. European Archives of Psychiatry and Clinical Neuroscience 250(4): 207-214.

\section{Acknowledgements}

Research was supported by the Deutsche Forschungsgemeinschaft. We thank Drs. K. Pröpster, H. Watzl, W. Höcker, A. Schiller, B. Schuller and P. Rössner for accomplishing the diagnostics and clinical status of the patients. 